Educación Física y Ciencia, abril-junio 2018, vol. 20, n² 2, e051. ISSN 1514-0105

Universidad Nacional de La Plata

Facultad de Humanidades y Ciencias de la Educación

Centro de Historia Argentina y Americana

\title{
Meninos e meninas obesas apresentam pior desempenho em tarefas motoras específicas
}

\author{
Raísa Carvalho da Silva \\ Universidade do Estado de Santa Catarina (UDESC), Brasil \\ rahisac@gmail.com \\ Diego Grasel Barbosa \\ Universidade do Estado de Santa Catarina (UDESC), Brasil \\ Fernando Luis Cardoso \\ Universidade do Estado de Santa Catarina (UDESC), Brasil \\ João Otacilio Libardoni dos Santos \\ Universidade Federal do Amazonas (UFAM), Brasil \\ Érico Pereira Gomes Felden \\ Universidade do Estado de Santa Catarina (UDESC), Brasil \\ Thaís Silva Beltrame \\ Universidade do Estado de Santa Catarina (UDESC), Brasil
}

Cita sugerida: Carvalho da Silva, R., Grasel Barbosa, D., Cardoso, F. L., Pereira domes Felden, E. y Thaís, S. B. (2018). Meninos e meninas obesas apresentam pior desempenho em tarefas motoras específicas. Educación Física y Ciencia, 20 (2), e051. https://doi.org/10.24215/1514-0105e051

Recibido: 09 agosto 2017 - Aceptado: 20 diciembre 2017 - Publicado: 27 abril 2018

(c) (1) (2) (2) Esta obra está bajo licencia Creative Commons Atribución-NoComercial-CompartirIgual 4.0 Internacional cc. 


\title{
Meninos e meninas obesas apresentam pior desempenho em tarefas motoras específicas
}

\author{
Obese boys and girls present worse performance in specific motor tasks \\ Muchachos y muchachas obesas presentan peor rendimiento en tareas motoras específicas \\ Raisa Carvalho da Silva \\ Universidade do Estado de Santa Catarina (UDESC), \\ Brasil \\ rahisac@gmail.com \\ Diego Grasel Barbosa \\ Universidade do Estado de Santa Catarina (UDESC), \\ Brasil \\ Fernando Luis Cardoso \\ Universidade do Estado de Santa Catarina (UDESC), \\ Brasil \\ João Otacilio Libardoni dos Santos \\ Universidade Federal do Amazonas (UFAM), Brasil \\ Érico Pereira Gomes Felden \\ Universidade do Estado de Santa Catarina (UDESC), \\ Brasil \\ Thais Silva Beltrame \\ Universidade do Estado de Santa Catarina (UDESC), \\ Brasil
}

\section{Resumo:}

Objetivou-se comparar o desempenho motor nas tarefas da bateria Körperkoordination Test für Kinder (KTK) de crianças com diferentes status de peso. Participaram do estudo, 350 crianças de oito a 10 anos. Investigaram-se: Índice de massa corporal (IMC), circunferência de cintura (CC), razão cintura-estatura (RCEst) e tarefas do KTK. Utilizou-se o teste qui-quadrado para verificar associações e o teste de Kruskal Wallis para comparação das pontuações das tarefas motoras entre os status de peso. Verificaramse que os meninos com sobrepeso e obesidade apresentaram piores resultados na tarefa de equilíbrio em marcha à retaguarda $(\mathrm{p}<0,001)$ e saltos monopedais $(\mathrm{p}<0,011)$, comparados aos meninos com baixo peso e eutróficos. Além disso, meninas obesas apresentaram piores resultados na tarefa de equilíbrio em marcha à retaguarda $(\mathrm{p}<0,001)$ comparadas às meninas com baixo peso, peso normal e sobrepeso. Conclui-se que crianças sobrepeso e obesidade apresentaram piores desempenhos nas tarefas do KTK comparadas às crianças eutróficas e com baixo peso.

Palavras-chave: Obesidade, Desempenho motor, Status de peso, Crianças.

\section{AbStract:}

The objective of this study was to compare the motor performance in the tasks of the Körperkoordination Test für Kinder (KTK) of children with different weight status. A total of 350 children aged 8 to 10 years old participated in the study. Body mass index (BMI), waist circumference (WC), waist-to-height ratio (KST) and KTK tasks were investigated. The chi-square test was used to verify associations and the Kruskal Wallis test was used to compare motor task scores between weight status. It was verified that overweight and obese boys had worse results in the task of balance in gait at the rear $(\mathrm{p}<0.001)$ and single-sheeled jumps $(\mathrm{p}$ $<0.011$ ), compared to boys with low weight and eutrophic. In addition, obese girls presented worse results in the task of balance in gait at the rear $(\mathrm{p}<0.001)$ compared to the girls with low weight, normal weight and overweight. It was concluded that overweight and obese children presented worse performances in KTK tasks compared to eutrophic and low weight children. 
KEYworDs: Obesity, Motor skills, Weight status, Children.

\section{INTRODUÇÃO}

Estudos têm demonstrado grande variação na prevalência de sobrepeso e obesidade na infância, com variações de 7,6\% a 44,4\% em diversos países (Cadenas-Sanchez et al., 2016; Greier, Riechelmann, \& Burtscher, 2014; Silveira, Barbosa, \& Vieira, 2015; Smetanina et al., 2015; M. C. C. d. Souza et al., 2014) . Além disso, o sobrepeso e obesidade são mais frequentemente observados nas meninas (Wang et al., 2007) e apresentam considerável impacto negativo no desempenho motor (Marramarco et al., 2012; Zanella, Bandeira, de Souza, \& Valentini, 2015).

A obesidade infantil, além de repercutir negativamente no desempenho motor, está associada a fatores genéticos hereditários (hiperfagia e disfunção hipotalâmica) (Waalen, 2014) e ao ambiente e estilo de vida sedentário. E isto pode implicar em sérios riscos para saúde física, bem estar e persistência do excesso de peso na vida adulta (Marramarco et al., 2012). Segundo Pelozin et al., (2010), o aumento dos níveis de obesidade na escola tem sido explicado pelos crescentes níveis de sedentarismo e inatividade física de crianças e adolescentes. O sedentarismo por sua vez, pode impactar negativamente na coordenação motora repercutindo no aumento do Índice de Massa Corporal (IMC) (Melo \& Lopes, 2013).

A relevância da relação entre o nível de competência motora e o status de peso em crianças e adolescentes tem sido demonstrada em inúmeros estudos (M. S. d. Souza \& Spessato, 2014; M. Souza, Spessato, \& Valentini, 2014; Zanella et al., 2015). Segundo Marramarco et al., (2012), o estado nutricional das crianças é um fator que está associado ao desempenho das habilidades motoras, indicando que crianças com excesso de peso e obesidade tendem a apresentar desempenho motor pobre ou muito pobre.

Neste ínterim, em estudo longitudinal de D'hondt et al., (2013), os autores verificaram que o status de peso aliado com a não participação de atividades esportivas, atuou como preditor negativo do nível de coordenação motora ao final de um período de dois anos. Além disto, Lopes et al. (2012), verificaram correlações negativas entre o nível de coordenação motora e o IMC em crianças de oito a dez anos de ambos os sexos e que aquelas com sobrepeso e obesidade, apresentaram piores desempenhos nas tarefas do Körperkoordination Test für Kinder (KTK) comparadas aos seus pares eutróficos.

Além da associação do status de peso e desempenho motor, pesquisadores têm associado à coordenação motora na infância com o envolvimento de práticas motoras (Haga, 2008; Santos, Ribeiro, Pellegrini, Rocha, \& Hiraga, 2012) e atividade física. A associação positiva entre estas variáveis pode melhorar o desempenho motor (Laukkanen, Pesola, Havu, Sääkslahti, \& Finni, 2014) e desempenho acadêmico de crianças (Ericsson \& Karlsson, 2014; Okuda \& Pinheiro, 2015).

Com relação aos testes que se propõem a mensurar o desempenho motor, o teste de coordenação corporal para crianças e adolescentes, KTK, tem sido o mais utilizado na literatura para avaliar a coordenação motora nesta população. Vários desses estudos destacam-se por associar o desempenho motor no KTK com o IMC (D'Hondt et al., 2014; Luz et al., 2015). No entanto, tem-se observado carência de estudos que possam identificar em quais tarefas crianças com sobrepeso e obesidade apresentam mais dificuldades.

Com a finalidade de compreender melhor o desempenho das habilidades e capacidades motoras em crianças com diferentes status de peso o presente estudo objetivou comparar o desempenho nas tarefas motoras da bateria KTK de crianças de oito a 10 anos com diferentes status de peso. 


\section{MÉTODO}

\section{Participantes do estudo}

Participaram do estudo 350 escolares com idades de oito a 10 anos, de ambos os sexos (164 meninos e 186 meninas). As escolas que fizeram parte da pesquisa foram selecionadas de acordo com a disponibilidade de participação das mesmas. Foram escolhidas três escolas, localizadas na cidade de Manaus-AM, sendo duas escolas públicas e uma escola privada. A amostragem foi do tipo não probabilística intencional. Os pais ou responsáveis das crianças foram informados do procedimento da pesquisa e assinaram o Termo de Consentimento Livre e Esclarecido - TCLE, autorizando assim a criança a participar. Como critérios de inclusão foram considerados: possuir idade de oito e dez anos; estar devidamente matriculado e frequentando a escola. Foram excluídas as crianças com deficiências física e/ou intelectuais verificados a partir de dados informados pelos professores e equipe diretiva da escola. O estudo foi aprovado pelo Comitê de Ética e Pesquisa em Seres Humanos da Universidade do Estado de Santa Catarina (UDESC), sob o parecer $n^{\circ}$ 159.196/2012, respeitando os preceitos da experimentação com seres humanos e todos os participantes permaneceram anônimos.

\section{Variáveis Investigadas}

Para a seleção das tarefas motoras utilizadas na pesquisa levou-se em consideração a elevada quantidade de estudos que utilizaram o instrumento Körperkoordination Test für Kinder (KTK), no cenário nacional e internacional (Bustamante, Lopes, da Silva, \& Ribeiro, 2010; D'Hondt et al., 2013; D’Hondt, Deforche, De Bourdeaudhuij, \& Lenoir, 2009)

O KTK abrange quatro testes: a) equilíbrio em marcha à retaguarda; b) saltos monopedais; c) saltos laterais; d) transposição lateral. O teste de coordenação engloba crianças e adolescentes de 5 a 14 anos de idade. Após a aplicação dos testes, o resultado de cada tarefa foi comparado com os valores normativos (tabelas elaboradas pelos autores do KTK). Para cada tarefa do teste é atribuído um quociente motor (QM), e a soma dos quatro quocientes motores gera o somatório dos quocientes motores (SQM). Este pode ser apresentado em percentil e em valores absolutos. A partir desses valores as crianças foram classificadas conforme sexo e idade nas seguintes categorias: (1) perturbações da coordenação ( $\mathrm{QM}<70)$; (2) insuficiência coordenativa (71 $\leq \mathrm{QM} \leq 85)$; (3) coordenação normal $(86 \leq \mathrm{QM} \leq 115)$; (4) coordenação boa $(116 \leq \mathrm{QM} \leq 130)$; (5) coordenação muito boa $(131 \leq \mathrm{QM} \leq 145)$. Para as análises foi utilizada apenas a pontuação do quociente motor $(\mathrm{QM})$ em cada tarefa.

Para a obtenção dos dados antropométricos os procedimentos foram padronizados seguindo as orientações de Alvarez e Pavan et al., (2003). Neste caso, as crianças usaram roupas adequadas para a prática de Educação Física, e não utilizaram calçados. Para a obtenção dos valores de massa corporal, foi utilizada balança digital da marca LAICA, com plataforma, escalonada em quilos e intervalos de 200 gramas, com registro mínimo de $12 \mathrm{~kg}$ e máximo de $136 \mathrm{~kg}$. A estatura foi mensurada utilizando uma fita métrica com escala de $0,1 \mathrm{~cm}$, fixada a parede sem rodapé. Para aferição da estatura o aluno foi posicionado em pé com o peso igualmente distribuído, tomando a posição ortostática. A partir dos dados antropométricos coletados foi determinado o índice de massa corporal $\left(\mathrm{IMC}=\right.$ massa corporal $(\mathrm{kg}) /$ estatura2 $\left.\left(\mathrm{m}^{2}\right)\right)$. O status de peso foi classificado por meio do IMC, no qual o critério utilizado foram os valores de referência propostos por Cole et al., (2000) e Cole et al.,(2007) que classifica em baixo peso, eutrófico, sobrepeso e obeso. 


\section{Tratamento de dados}

A análise dos dados foi feita a partir do programa estatístico Statistical Package for Social Sciences (SPSS) versão 20.0. Para análise descritiva foram calculadas as médias, desvios-padrões e distribuição de frequências. Para verificar a normalidade dos dados foi utilizado o teste de Kolmogorov-Smirnov. Os dados não apresentaram distribuição normal e em virtude disto, recorreram-se a análises não paramétricas.

Em relação à estatística inferencial, para a associação das variáveis categóricas: sexo e status de peso foi utilizado o teste de associação qui-quadrado. Utilizou-se o teste U de Mann Whitney para verificar as diferenças das variáveis numéricas de idade, IMC e pontuações das tarefas do KTK entre meninos e meninas. Para verificar a diferença entre médias nas tarefas do KTK e status de peso (baixo peso, eutrófico, sobrepeso e obeso) utilizou-se o teste de Kruskal- Wallis. Foi adotado intervalo $95 \%$ de confiança $(p<0,05)$.

\section{RESULTADOS}

$\mathrm{Na}$ Tabela 1 foram apresentadas as estatísticas descritivas (média, desvios-padrões ou percentuais) das variáveis avaliadas. A média de idade dos participantes foi 8,94 (0,78) anos. Os meninos apresentaram médias superiores às meninas na pontuação total do KTK $(\mathrm{p}<0,001)$. Nas tarefas de saltos monopedais, saltos laterais e transposição lateral, as meninas obtiveram pior desempenho comparadas aos meninos $(\mathrm{p}<0,001)$. $\mathrm{Na}$ amostra geral, 16,3\% das crianças apresentavam sobrepeso e $8,0 \%$ obesidade.

\begin{tabular}{lcccc} 
Tabela l. Dados descritivos da amostra. & \multicolumn{1}{l}{} \\
\hline \multicolumn{1}{c}{ Variável } & Geral & Masculino & Feminino & p-valor* \\
\hline Idade, anos & $8,94(0,78)$ & $8,99(0,79)$ & $8,90(0,77)$ & 0,317 \\
KTK, escore padrão final & $95,50(12,32)$ & $99,83(9,59)$ & $91,69(13,20)$ & $<0,001$ \\
Equilíbrio em marcha à retaguarda, & $93,72(12,14)$ & $93,29(12,00)$ & $94,10(12,28)$ & 0,674 \\
QM & $60,39(11,63)$ & $64,19(10,93)$ & $57,04(11,21)$ & $<0,001$ \\
Saltos monopedais, QM & $76,96(16,18)$ & $84,59(13,51)$ & $70,22(15,35)$ & $<0,001$ \\
Saltos laterais, QM & $75,86(13,74)$ & $79,12(13,20)$ & $72,99(13,59)$ & $<0,001$ \\
Transposição lateral, QM & & & & \\
Status de peso, \% & 10,6 & 8,5 & 12,4 & \\
$\quad$ Baixo peso & 65,1 & 68,9 & 61,8 & 0,519 \\
$\quad$ Eutrófico & 16,3 & 15,2 & 17,2 & \\
$\quad$ Sobrepeso & 8,0 & 7,3 & 8,6 & \\
$\quad$ Obeso & 8,0 &
\end{tabular}

TABELA 1

Dados descritivos da amostra.

$\mathrm{Na}$ Tabela 2, por meio da análise de Dunn, foi verificado que as crianças obesas apresentaram menores pontuações nas tarefas de marcha à retaguarda e saltos monopedais quando comparadas às crianças eutróficas e com baixo peso $(\mathrm{p}<0,001)$.

\begin{tabular}{|c|c|c|c|c|c|}
\hline KTK, tarefas & Baixo peso & Eutrófico & Sobrepeso & Obeso & p-valor \\
\hline $\begin{array}{l}\text { Equilíbrio em marcha à } \\
\text { retaguarda }\end{array}$ & $98,38(12,64)^{a}$ & $95,21(11,19)^{\mathrm{a}}$ & $90,70(10,81)^{a b}$ & $81,61(13,30)^{b}$ & $<0,001$ \\
\hline Saltos monopedais & $60,4(11,45)^{\mathrm{a}}$ & $61,83(11,65)^{\mathrm{a}}$ & $58,54(11,63)^{a b}$ & $52,64(8,01)^{b}$ & $<0,001$ \\
\hline Saltos laterais & $77,57(15,26)^{\mathrm{a}}$ & $77,60(15,91)^{\mathrm{s}}$ & $75,84(18,01)^{\mathrm{a}}$ & $73,11(15,85)^{\mathrm{a}}$ & 0,359 \\
\hline Transposição lateral & $78,11(10,54)^{\mathrm{a}}$ & $76,23(13,38)^{\mathrm{a}}$ & $74,30(17,77)^{\mathrm{a}}$ & $73,07(10,53)^{\mathrm{a}}$ & 0,315 \\
\hline
\end{tabular}

TABELA 2

Comparação das tarefas do KTK entre os status de peso na amostra geral 
De acordo com a Tabela 3, verificaram-se diferenças significativas em algumas tarefas do KTK tanto para o sexo masculino como para o sexo feminino. Os meninos obesos apresentaram menores pontuações nas tarefas de equilíbrio em marcha à retaguarda $(\mathrm{p}=0,001)$ e saltos monopedais $(\mathrm{p}<0,001)$ quando comparados aos meninos com baixo peso, eutróficos e com sobrepeso. Com relação ao sexo feminino, verificou-se que as meninas obesas apresentaram menores pontuações na tarefa de equilíbrio em marcha à retaguarda comparadas as meninas com baixo peso e eutróficas $(\mathrm{p}<0,001)$.

\begin{tabular}{lccccc}
\multicolumn{6}{c}{ Tabela 3. Comparação das tarefas do KTK entre os status de peso no sexo masculino e feminino } \\
\cline { 2 - 6 } KTK, tarefas & \multicolumn{5}{c}{ Sexo masculino } \\
\cline { 2 - 6 } & Baixo peso & Eutrófico & Sobrepeso & Obeso & p-valor \\
\hline Equilíbrio em marcha à & $98,36(10,40)^{\mathrm{a}}$ & $94,28(10,89)^{\mathrm{a}}$ & $93,96(10,78)^{\mathrm{a}}$ & $76,58(14,00)^{\mathrm{b}}$ & 0,001 \\
retaguarda & $68,00(10,26)^{\mathrm{a}}$ & $65,40(10,79)^{\mathrm{a}}$ & $61,56(11,10)^{\mathrm{a} b}$ & $53,83(5,11)^{\mathrm{b}}$ & $<0,001$ \\
Saltos monopedais & $87,00(14,31)^{\mathrm{a}}$ & $83,99(13,71)^{\mathrm{a}}$ & $86,76(11,30)^{\mathrm{a}}$ & $83,83(15,70)^{\mathrm{a}}$ & 0,663 \\
Saltos laterais & $80,00(13,58)^{\mathrm{a}}$ & $79,34(12,67)^{\mathrm{a}}$ & $79,20(16,49)^{\mathrm{a}}$ & $75,92(11,19)^{\mathrm{a}}$ & 0,834 \\
Transposição lateral & \multicolumn{5}{c}{ Sexo feminino } \\
\hline \multicolumn{1}{c}{ KTK, tarefas } & Baixo peso & Eutrófico & Sobrepeso & Obeso & $\mathrm{p}$-valor \\
\hline Equilíbrio em marcha à & $98,39(14,06)^{\mathrm{a}}$ & $96,11(11,44)^{\mathrm{a}}$ & $88,16(10,31)^{\mathrm{b}}$ & $85,37(11,80)^{\mathrm{b}}$ & $<0,001$ \\
retaguarda & $55,52(9,52)^{\mathrm{a}}$ & $58,32(11,44)^{\mathrm{a}}$ & $56,19(11,66)^{\mathrm{a}}$ & $51,75(9,71)^{\mathrm{a}}$ & 0,124 \\
Saltos monopedais & $71,83(13,00)^{\mathrm{a}}$ & $71,31(15,45)^{\mathrm{a}}$ & $67,31(17,77)^{\mathrm{a}}$ & $65,81(11,80)^{\mathrm{a}}$ & 0,316 \\
Saltos laterais & $76,96(8,32)^{\mathrm{a}}$ & $73,18(13,40)^{\mathrm{a}}$ & $70,47(18,05)^{\mathrm{a}}$ & $70,94(9,82)^{\mathrm{a}}$ & 0,358 \\
Transposição lateral & \multicolumn{3}{c}{} &
\end{tabular}

\section{TABELA 3}

Comparação das tarefas do KTK entre os status de peso no sexo masculino e feminino

Além disso, foi verificado que à medida que o IMC aumentava, menores eram as pontuações das crianças nas tarefas de marcha à retaguarda $(\mathrm{r}=-0,271 \mathrm{p}<0,001)$.

\section{DISCUSSÃO}

A proposta do estudo foi comparar o desempenho nas tarefas motoras da bateria KTK de crianças de oito a 10 anos com diferentes status de peso. Os principais achados do presente estudo demonstraram que meninos e meninas obesos apresentam de forma geral baixo desempenho motor em tarefas específicas da bateria.

Os resultados encontrados com o quociente motor de cada tarefa do KTK demonstraram que nos meninos, as pontuações das tarefas de equilíbrio em marcha à retaguarda e saltos monopedais foram diferentes entre os status de peso, sendo que os meninos obesos obtiveram pontuação menor do que os eutróficos e com baixo peso. Nas análises das meninas apenas na tarefa de equilíbrio em marcha à retaguarda foi verificada diferença considerando o status de peso, sendo que os piores resultados foram verificados nas meninas com sobrepeso e obesidade.

Esses resultados podem ter interferência de uma série de mudanças ocasionadas por características antropométricas, estruturais e de composição corporal do ser humano, durante a vida. Essas mudanças se relacionam de forma direta com o desempenho motor, sendo influenciadas por experiências vividas, como oportunidade e interesses diferenciados entre meninos e meninas, especificidades individuais e complexidade de tarefas (Camargo \& Pereira, 2012).

Segundo Camargo e Pereira, (2012) crianças obesas apresentam geralmente má postura e produzem maior tensão sobre as estruturas e a base de suporte, e ainda, apresentam uma maior concentração de gordura na região abdominal, deslocando o centro de gravidade do corpo para frente, acarretando em aumento da lordose e anteversão pélvica, fatores estes, responsáveis pela ocorrência de problemas no equilíbrio (Aleixo, Guimarães, de Walsh \& Pereira, 2012). Além da explicação anatomo-funcional, déficits de equilíbrio em crianças com excesso de peso ou obesas podem ser devido à baixa participação de atividades que envolvam esta e outras capacidades físicas relacionadas à baixa autoestima e sentimentos de inferioridade motora perante seus eutróficos (Spessato, Gabbard, Robinson, \& Valentini, 2013). 
$\mathrm{Na}$ análise geral (sexo masculino e feminino) as crianças com sobrepeso e obesidade apresentaram dificuldades significativas apenas nas tarefas de equilíbrio em marcha à retaguarda e saltos monopedais. Estes resultados podem relacionar-se com a natureza das tarefas. Essas tarefas exigem equilíbrio e deslocamento, podendo ser influenciadas pelo centro de gravidade. Corroborando com esses resultados Lopes et al., (2012), verificaram que crianças com alto IMC apresentaram menores pontuações nas tarefas do KTK.

No estudo de Deforche et al., (2009), os autores verificaram que meninos com idades de oito a 10 anos com sobrepeso, demonstraram menor capacidade em testes de equilíbrio estático e dinâmico quando comparados com seus pares de peso normal. Além disto, D’hondt et al., (2009), também verificaram correlação inversa entre coordenação motora e IMC em crianças de cinco a 10 anos na tarefa de equilíbrio $(r=-0,20)$ (estático e dinâmico) e habilidades com bola $(r=-0,46)$ com escore $\mathrm{z}$ do IMC ajustado para sexo e idade. De forma similar D'hondt et al., (2008), encontraram que crianças de cinco a 12 anos com sobrepeso e obesidade apresentaram maiores prejuízos nos testes de controle e organização postural do que crianças eutróficas. Estes resultados reforçam as diferenças encontras no presente estudo relacionadas à maior dificuldade de equilíbrio estático e dinâmico em crianças com sobrepeso e obesidade. Deste modo, supõe-se que alternativas para reverter este quadro estão intrinsecamente ligadas à intervenção motora especifica e melhora nos níveis de atividade física para a perda de peso e consequentemente uma possível melhora do equilíbrio destas crianças.

O fato de crianças com sobrepeso e obesidade apresentarem piores desempenhos em tarefas específicas pode ser justificado pela influência de hábitos sedentários nessas crianças e consequentemente reduzidos níveis de desempenho motor (Melo \& Lopes, 2013).

A limitação relacionada a presente pesquisa está em não ter sido realizado cálculo amostral a fim de se buscar representatividade da população investigada. Sugerem-se novos estudos que investiguem a relação das tarefas com outros indicadores antropométricos e estudos de cortes longitudinais buscando entender melhor a relação entre essas variáveis.

\section{CONCLUSÃO}

Conclui-se que crianças com sobrepeso e obesidade apresentaram piores desempenhos nas tarefas de equilíbrio em marcha à retaguarda e saltos monopedais da bateria KTK, quando comparadas com seus homólogos eutróficos e com baixo peso. É, portanto necessário que os professores de educação física que atuam com esta população possam entender a importância de oportunizar mais experiências motoras para as crianças com alguma dificuldade devido seu maior IMC. Estas crianças podem apresentar piores desempenhos em determinadas tarefas, sendo papel do professor de educação física auxiliar no desenvolvimento dessas crianças, juntamente com os pais e a escola. No sentido de que a tríade (professor de educação física, pais e a escola) possa trabalhar juntos para reverter os casos de crianças com sobrepeso e obesidade.

\section{REFERÊNCIAS}

Aleixo, A. A., Guimarães, E. L., de Walsh, I. A. P., Pereira, K. (2012). Influência do sobrepeso e da obesidade na postura, na praxia global e no equilíbrio de escolares. Journal of Human Growth and Development, 22(2), 239-245.

Alvarez, B. R., Pavan, A. L., \& Petroski, E.L. (2003). Alturas e comprimentos. Antropometria: técnicas e padronizações, 2, 59-71.

Bustamante, A., Lopes, V. P., da Silva, R. M. G. \& Ribeiro, J. A. (2010). Modelação longitudinal dos níveis de coordenação motora de crianças dos seis aos 10 anos de idade da Região Autônoma dos Açores, Portugal. Rev. bras. Educ. Fís. Esporte, 24(2), 259-273. 
Cadenas-Sanchez, C., Nyström, C., Sanchez-Delgado, G., Martinez-Tellez, B., Mora-Gonzalez, J., Risinger, AS., Löf, M. (2016). Prevalence of overweight/obesity and fitness level in preschool children from the north compared with the south of Europe: An exploration with two countries. Pediatric obesity, 11(5) 403-410.

Camargo, C. S. \& Pereira, K. (2012). Evolução antropométrica, postural e do equilíbrio de crianças com sobrepeso e obesidade. ConScientiae Saúde, 11(2), 256-264.

Cole, T. J., Bellizzi, M. C., Flegal, K. M., \& Dietz, W.H. (2000). Establishing a standard definition for child overweight and obesity worldwide: international survey. Bmj, 320(7244), 1240.

Cole, T. J., Flegal, K. M., Nicholls, D., \& Jackson, A. A. (2007). Body mass index cut offs to define thinness in children and adolescents: international survey. Bmj, 335(7612), 194.

D'Hondt, E., Deforche, B., Gentier, I., De Bourdeaudhuij, I., Vaeyens, R., Philippaerts, R., \& Lenoir, M.. (2013). A longitudinal analysis of gross motor coordination in overweight and obese children versus normal-weight peers. International journal of obesity, 37(1), 61-67.

D'Hondt, E., Deforche, B., Gentier, I., Verstuyf, J., Vaeyens, R., Bourdeaudhuij, I., Lenoir, M. (2014). A longitudinal study of gross motor coordination and weight status in children. Obesity, 22(6), 1505-1511.

D’Hondt, E., Deforche, B., De Bourdeaudhuij, I., \& Lenoir, M. (2008). Childhood obesity affects fine motor skill performance under different postural constraints. Neuroscience letters, 440(1), 72-75.

D'Hondt, E., Deforche, B., De Bourdeaudhuij, I., \& Lenoir, M. (2009). Relationship between motor skill and body mass index in 5-to 10-year-old children. Adapted Physical Activity Quarterly, 26(1), 21-37.

Deforche, B. I., Hills, A. P., Worringham, C. J., Davies, P. S.W., Murphy, A. J., Bouckaert, J. J., \& De Bourdeaudhuij, I. M. (2009). Balance and postural skills in normal-weight and overweight prepubertal boys. International Journal of Pediatric Obesity, 4(3), 175-182.

Ericsson, I., \& Karlsson, M. K. (2014). Motor skills and school performance in children with daily physical education in school-a 9-year intervention study. Scandinavian journal of medicine \& science in sports, 24(2), 273-278.

Greier, K., Riechelmann, H. \& Burtscher, M. (2014). Prevalence of obesity and motor performance capabilities in Tyrolean preschool children. Wiener klinische Wochenschrift, 126(13-14), 409-415.

Haga, M. (2008). The relationship between physical fitness and motor competence in children. Child: care, health and development, 34(3), 329-334.

Laukkanen, A., Pesola, A., Havu, M., Sääkslahti, A., \& Finni, T. (2014). Relationship between habitual physical activity and gross motor skills is multifaceted in 5-to 8-year-old children. Scandinavian journal of medicine \& science in sports, 24(2), e102-e110.

Lopes, V. P., Stodden, D. F., Bianchi, M. M., Maia, J. A. R., \& Rodrigues, L. P. (2012). Correlation between BMI and motor coordination in children. Journal of Science and Medicine in Sport, 15(1), 38-43.

Luz De Oliveira, L.G., Seabra, T. E, Filipe, A., Santos., R., Padez., C. \& Ferreira, J. P. (2015). Associação entre IMC e teste de coordenação corporal para crianças (KTK). Uma meta-análise.

Marramarco, C. A., Krebs, R. J., Valentini, N. C., da Silva Ramalho., M. H., dos Santos., J. O. L., \& Nobre, G. C. (2012). Crianças desnutridas pregressas, com sobrepeso e obesas apresentam desempenho motor pobre. Journal of Physical Education, 23(2), 175-182. doi: 10.4025/reveducfis. v23i2. 13002.

Melo, M. M., \& Lopes, V. P. (2013). Associação entre o índice de massa corporal e a coordenação motora em crianças. Rev Bras Educ Fís Esporte, 27(1), 7-13.

Okuda, P. M. M. \& Pinheiro, F. H. (2015). Motor performance of students with learning difficulties. Procedia-Social and Behavioral Sciences, 174, 1330-1338.

Pelozin, F., Folle, A., Collet, C., Botti, M., \& do Nascimento, J. V. (2010). Nível de coordenação motora de escolares de 9 a 11 anos da Rede Estadual de Ensino da cidade de Florianópolis-SC. Revista Mackenzie de Educação Física e Esporte, 8(2).

Santos, M M. D. A., Ribeiro, S. M. L., Pellegrini, A. M., Rocha, P. R. H. \& Hiraga, C. Y. (2012). Crianças com dificuldades motoras apresentam baixos níveis de aptidão física? Motriz: Revista de Educação Física, 18(4) 748-756. 
Silveira, F. J. F., Barbosa, J. C. \& Vieira, V. A. M. (2015). Prevalence of overweight and obesity in children of four state schools in Belo Horizonte, Minas Gerais.

Smetanina, N., Albaviciute, E., Babinska, V., Karinauskiene, L., Albertsson-Wikland, K., Petrauskiene, A., \& Verkauskiene, R. (2015). Prevalence of overweight/obesity in relation to dietary habits and lifestyle among 717 years old children and adolescents in Lithuania. BMC public health, 15(1), 1001.

Souza, M. C. C., Tibúrcio, J. D., Bicalho, J. M. F., Rennó, H. M. S., Dutra, J. S., Campos, L. G., \& Silva, E. S. (2014). Factors associated with obesity and overweight in school-aged children. Texto \& Contexto - Enfermagem, 23, 712-719.

Souza, M. S. \& Spessato, B. C. (2014). Percepção de competência motora e índice de massa corporal influenciam os níveis de atividade física? Revista Brasileira de Ciência e Movimento, 22(2), 78-86. DOI: http:// dx.doi.org/10.18511/0103-1716/rbcm.v22n2p78-86..

Souza, M. S., Spessato, B. S., \& Valentini,. N. C. (2015). Habilidades motoras fundamentais e as possíveis relações com níveis de atividade física, estado nutricional e sexo. Rev Acta Bras Mov Hum, 4(1), 41-51.

Spessato, B. C., Gabbard, C., Robinson, L, \& Valentini, N.C. (2013). Body mass index, perceived and actual physical competence: the relationship among young children. Child: care, health and development, 39(6), 845-850.

Waalen, J. (2014). The genetics of human obesity. Translational Research, 164(4), 293-301.

Wang, Y., Liang, H., Tussing, L., Braunschweig, C., Caballero, B. \& Flay, B. (2007). Obesity and related risk factors among low socio-economic status minority students in Chicago. Public health nutrition, 10(09), 927-938.

Zanella, L. W., Bandeira, P. F. R., de Souza, M. S. \& Valentini, N. C. (2015). Há associação entre o desempenho motor e estado nutricional de escolares? Conexões, 13(2), 136-148. 